\title{
dc-field-induced enhancement and inhibition of spontaneous emission in a cavity
}

\author{
G. S. Agarwal and P. K. Pathak \\ Physical Research Laboratory, Navrangpura, Ahmedabad-380009, India
}

(Received 28 December 2003; published 20 August 2004)

\begin{abstract}
We demonstrate how spontaneous emission in a cavity can be controlled by the application of a dc field. The method is especially suitable for Rydberg atoms. We present a simple argument based on Stark shifts for the control of emission.
\end{abstract}

DOI: 10.1103/PhysRevA.70.025802

PACS number(s): 42.50.Lc, 42.50.Ct, 42.50.Hz

The manipulation of spontaneous emission has been extensively studied. Methods involving either external fields $[1-8]$ or appropriate cavities $[9,10]$ have been suggested. The use of external fields enables one to control spontaneous emission via quantum interference effects [5,6]. Purcell [11] recognized how the emission rate in a single mode cavity can be much higher than in free space. Several demonstrations of the cavity-enhanced spontaneous rates exist in the literature $[9,10]$. Kleppner [12] discovered that the radiation rate in a cavity can be inhibited by choosing the transition frequency, such that the density of states at this frequency is insignificant. Quantum interference between various channels $[3,4,6,7]$ could also result in the inhibition of emission. Further, very interesting, experiments $[1,2,6-8]$ on the fieldinduced inhibition of emission in a cavity were reported. In these experiments the applied fields were resonant with atomic transitions. In this paper we show how a possible control of spontaneous emission can be obtained by using dc fields. We treat the case of atoms in a cavity and explain in rather simple terms the origin of the control produced by the dc fields. To be precise we are considering only the effect of dc field on the part of decay, which is due to the emission in the cavity mode. The decay of the atom depends on the detuning between the atomic frequency and the cavity frequency. The application of the dc field makes the detuning dependent on the field (Stark effect) and thus the dc field provides a control of the spontaneous emission. The dc-fieldinduced modification of spontaneous emission in free space is treated in Ref. [3].

We next describe how to calculate the dc-field-induced modification of the decay characteristics in a cavity. For our purpose we consider a two-level atom placed in a cavity, and a dc field (or low-frequency field) is injected inside the cavity. The Hamiltonian of the system can be written as

$$
H=\hbar \omega_{0} S^{z}+\hbar \omega_{c} a^{\dagger} a+\hbar g\left(a S^{+}+S^{-} a^{\dagger}\right)+\hbar \mathcal{E} \cos \Omega t\left(S^{+}+S^{-}\right),
$$

where $\omega_{0}$ is the atomic transition frequency, $\omega_{c}$ is the cavity mode frequency, and $g$ is the atom-cavity coupling constant. The term $\mathcal{E} \cos \Omega t$ corresponds to a low-frequency field, if $\Omega$ is chosen to be very small. Note that $\mathcal{E}$ has dimensions of frequency. The cavity field has been expressed in terms of annihilation and creation operators $a, a^{\dagger}$ and $S^{+}, S^{-}, S^{z}$ are usual atomic spin operators. We perform master equation calculations for the atom-cavity system. The density matrix of the system $\rho$ will evolve as

$$
\dot{\rho}=-\frac{i}{\hbar}[H, \rho]-\kappa\left(a^{\dagger} a \rho-2 a \rho a^{\dagger}+\rho a^{\dagger} a\right),
$$

where $2 \kappa$ gives the leakage of photons. It is related to the cavity $Q$ via $\kappa=\omega_{c} / 2 Q$. We will work in a frame rotating with atomic frequency $\omega_{0}$. The density matrix in this frame is given by

$$
\widetilde{\rho}=e^{i \omega_{0}\left(S^{z}+a^{\dagger} a\right) t / \hbar} \rho e^{-i \omega_{0}\left(S^{z}+a^{\dagger} a\right) t / \hbar} .
$$

Using Eqs. (2) and (3) we obtain the equation for $\widetilde{\rho}$,

$$
\dot{\tilde{\rho}}=-\frac{i}{\hbar}\left[H_{a}, \tilde{\rho}\right]-\kappa\left(a^{\dagger} a \tilde{\rho}-2 a \tilde{\rho} a^{\dagger}+\widetilde{\rho} a^{\dagger} a\right)-\frac{i}{\hbar}\left[H_{d}, \widetilde{\rho}\right],
$$

where

$$
\begin{gathered}
H_{a}=-\hbar \Delta a^{\dagger} a+\hbar g\left(a S^{+}+S^{-} a^{\dagger}\right), \\
H_{d}=\hbar \frac{\mathcal{E}}{2}\left\{S^{+}\left(e^{i\left(\omega_{0}+\Omega\right) t}+e^{i\left(\omega_{0}-\Omega\right) t}\right)+S^{-}\left(e^{-i\left(\omega_{0}+\Omega\right) t}+e^{-i\left(\omega_{0}-\Omega\right) t}\right)\right\},
\end{gathered}
$$

and $\Delta=\omega_{0}-\omega_{c}$ is the detuning. We first note that the experiments of Lange and Walther correspond to using a microwave field, and thus $\Omega \sim \omega_{0}$. The results of Purcell and Kleppner also follow from the master equation (4). For $\mathcal{E}=0$ and $g \ll \kappa$, we can derive an equation for the atomic density matrix $\tilde{\rho}_{a}$,

$$
\tilde{\rho}_{a}=\operatorname{Tr}_{c} \tilde{\rho},
$$

where $\operatorname{Tr}_{c}$ is trace over cavity field, by adiabatically eliminating cavity variables. This leads to

$$
\dot{\tilde{\rho}}_{a}=-i\left[\delta_{0} S^{z}, \tilde{\rho}_{a}\right]-\Gamma_{0}\left(S^{+} S^{-} \tilde{\rho}_{a}-2 S^{-} \tilde{\rho}_{a} S^{+}+\tilde{\rho}_{a} S^{+} S^{-}\right),
$$

where

$$
\Gamma_{0}=\frac{g^{2} \kappa}{\kappa^{2}+\Delta^{2}}, \delta_{0}=\frac{g^{2} \Delta}{\kappa^{2}+\Delta^{2}} .
$$

For resonant cavity $\omega_{c}=\omega_{0}, \delta_{0}=0$ and the decay rate $\Gamma_{0}$ $=g^{2} / \kappa$. There is cavity-induced enhancement if $g^{2} / \kappa$ is greater than the free space decay rate. Note that as the cavity is detuned $(\Delta \neq 0) \Gamma_{0}$ decreases, which is Kleppner's result for a single mode cavity. Experimental observation of the Purcell effect was made by Goy et al. [9]. Next, we investigate the effect of the applied dc or low-frequency field. Note that the last term in the master equation (4) is highly oscil- 


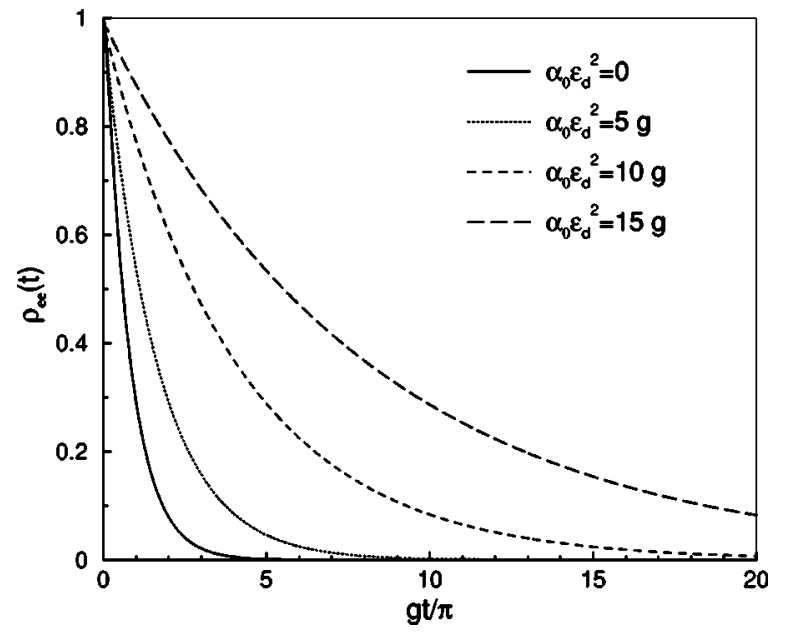

FIG. 1. The probability of the atom remaining in its excited state, $\rho_{e e} \equiv\langle e, 0|\rho| e, 0\rangle$ vs time, for $\kappa=5 g, \Delta=0, \Omega=0$, and for the different values of the dc field $\mathcal{E}_{d}$.

lating. We do time averaging for this, as such terms oscillating at the cavity frequency would not be normally observed. The time averaging is well justified here, as all other relevant time scales $g^{-1}, \kappa^{-1}, \Delta^{-1}$ are much larger than $\left(\omega_{0} \pm \Omega\right)^{-1}$. The inequality $\omega_{0} \gg g, \kappa, \Delta$ enables us to do the time averaging in a much simpler fashion, i.e., we can essentially ignore the terms having $H_{a}$ and $\kappa$ in (4). We relegate the details of time averaging to the Appendix. The calculation leads to the following time-averaged master equation:

$$
\begin{aligned}
\dot{\tilde{\rho}}= & i\left[\Delta_{e} a^{\dagger} a, \widetilde{\rho}\right]-i g\left[\left(a S^{+}+S^{-} a^{\dagger}\right), \tilde{\rho}\right]-\kappa\left(a^{\dagger} a \widetilde{\rho}-2 a \widetilde{\rho} a^{\dagger}\right. \\
& \left.+\widetilde{\rho} a^{\dagger} a\right),
\end{aligned}
$$

where

$$
\Delta_{e}=\Delta+2 \omega_{0} \mathcal{E}^{2} /\left(\omega_{0}^{2}-\Omega^{2}\right) .
$$

We note that the dc field contributes to the Stark shift of the two levels in question. We further note that these two atomic levels can also be shifted because of the interaction of the dc field with other levels. These can be accounted for by introducing the polarizabilities $\alpha_{e}$ and $\alpha_{g}$ of the levels $|e\rangle$ and $|g\rangle$ $[13,14]$. We can rewrite Eq. (10) as

$$
\Delta_{e}=\Delta+\alpha_{0} \mathcal{E}_{d}^{2}, \alpha_{0}=\alpha_{e}-\alpha_{g},
$$

where $\mathcal{E}_{d}$ is now the dc field in esu. The formulation of the Appendix can also be used to produce the well-known expressions for the $\alpha^{\prime s}$. The value of $\alpha_{0}$ is known for many low-lying, as well as Rydberg, transitions. The values of $\alpha_{0}$ have been calculated in the literature by converting infinite sums into the solution of differential equations.

Equation (9) can be solved, assuming that the atom is initially excited and the cavity field is in vacuum state. Equation (9) can be converted into a set of coupled equations in terms of the states $|e, 0\rangle,|g, 1\rangle$, and $|g, 0\rangle$. The results of the numerical integration are shown in Fig. 1 for different values of the parameter $\Delta_{e}$. Clearly there is inhibition as $\Delta_{e}$ increases. The effective detuning $\Delta_{e}$ changes due to the applied dc field. For a fixed cavity detuning $\Delta$ the dc field can make $\Delta_{e}$ larger or smaller depending on the sign of $\Delta$. The results can be understood by deriving analytical results in the bad cavity limit [15] $g \ll \kappa$ (and more precisely $g^{2} \ll \kappa^{2}+\Delta_{e}^{2}$ ). In this limit we can obtain a simpler equation for the atomic density matrix $\tilde{\rho}_{a}$, defined by Eq. (6). The final result for the atomic system is

$$
\dot{\tilde{\rho}}_{a}=-i\left[\delta_{e} S^{z}, \widetilde{\rho}_{a}\right]-\Gamma_{e}\left(S^{+} S^{-} \widetilde{\rho}_{a}-2 S^{-} \widetilde{\rho}_{a} S^{+}+\widetilde{\rho}_{a} S^{+} S^{-}\right),
$$

where

$$
\Gamma_{e}=\frac{g^{2} \kappa}{\kappa^{2}+\Delta_{e}^{2}}, \delta_{e}=\frac{g^{2} \Delta_{e}}{\kappa^{2}+\Delta_{e}^{2}} .
$$

Here, $\Gamma_{e}$ is the dc-field-modified decay parameter and $\delta_{e}$ is the net frequency shift. The ratio $\eta$ of the decays in the presence and absence of dc field is given by

$$
\eta=\frac{\Gamma_{e}}{\Gamma_{0}}=\frac{\kappa^{2}+\Delta^{2}}{\kappa^{2}+\Delta_{e}^{2}} .
$$

Clearly the dc field modifies the decay rate, which depends on the detuning. For the cavity resonant to the atomic transition $(\Delta=0)$, using Eq. (10), $\eta$ reduces to

$$
\eta=\frac{\kappa^{2}}{\kappa^{2}+\alpha_{0}^{2} \mathcal{E}_{d}^{4}} \approx\left(1+\frac{4 \mathcal{E}^{4}}{\kappa^{2} \omega_{0}^{2}}\right)^{-1} \text {, for } \Omega=0 .
$$

It is clear from Eq. (15) that the dc field inhibits the decay rate. Note that the inhibition starts becoming significant for

$$
\alpha_{0} \mathcal{E}_{d}^{2} \sim \kappa .
$$

Let us estimate the condition (16) for the Na Rydberg transition $23 S_{1 / 2} \rightarrow 22 P_{3 / 2}$, whose frequency is $340 \mathrm{GHz}$. For the sake of argument, we also assume $\alpha_{0} \mathcal{E}_{d}^{2} \sim 2 \mathcal{E}^{2} / \omega_{0}$. This transition has a dipole moment $d \sim 10^{-15}$ esu. The atom is placed in the cavity having one mode resonant to the atomic transition. Let us choose the cavity decay rate $\kappa=1 \mathrm{MHz}$. The condition (16) then leads to a Rabi frequency $\mathcal{E}$ of the order $400 \mathrm{MHz}$, which in turn, requires a dc field of the order of $10^{-2}$ esu. We note that the required dc field is small enough, so that the perturbative results for the Stark shift hold. We further note that the scalar and tensor polarizabilities are available for some $S$ and $P$ levels of $\mathrm{Na}[13,14]$, although the absolute values for both the $23 S_{1 / 2}$ and $22 P_{3 / 2}$ level are not available in Fabre et al. [13]. However, the reported polarizabilities for say, the $23 P$ level, are of the order of a few $\mathrm{MHz} /(\mathrm{Volt} / \mathrm{cm})^{2}$. Thus the condition (16) is realistic, and our finding that the dc field can be used to control spontaneous emission, can be implemented by the appropriate choice of the Rydberg transitions, [cf. the condition (16)]. We emphasize that we are discussing the inhibition or enhancement of spontaneous emission on a given transition, which is resonant with the cavity. This, for example, is the transition $23 S \rightarrow 22 P$ in the experiments of Goy et al. [9]. The authors of Ref. [9] emphasize this, as well, and it is in the spirit of the suggestion of Purcell [11]. It must be noted that the fieldionization techniques enable one to study transitions selectively [16].

In the case of cavities detuned from the atomic transition, spontaneous decay is smaller and the decay rate is given by 


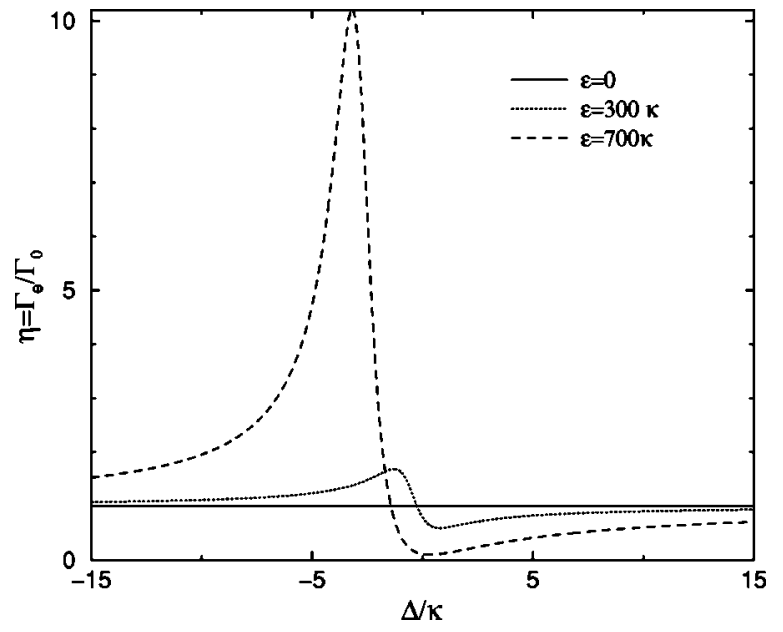

FIG. 2. The ratio $(\eta)$ of the decays in the presence and the absence of a dc field vs $\Delta / \kappa$. The parameters are $\omega_{0}=3.4 \times 10^{5} \kappa$ and $\Omega=0$.

$\Gamma=g^{2} \kappa /\left(\kappa^{2}+\Delta^{2}\right)$. Further inhibition of the decay rate is possible by applying a dc field. When a cavity is tuned below the atomic transition frequency ( $\Delta$ is positive), then there is a significant inhibition of spontaneous decay, which increases further as the applied dc field is increased. On the other hand, when a cavity is tuned above the atomic frequency ( $\Delta$ is negative), there is an enhancement in the atomic decay, i.e., on increasing the value of an applied dc field, the atom decays faster.

In Fig. 2 we show the behavior of the factor $\eta$ as a function of $\Delta$ for different values of the dc field. The enhancement, as well as the inhibition of spontaneous decay, occurs, depending on whether the cavity is tuned above or below the atomic frequency. The results shown in Fig. 2 are consistent with the results obtained by direct solution of Eq. (9).

In conclusion, we find that in the presence of a dc field, spontaneous emission can be inhibited significantly in the case of cavities resonant to atomic transition. In the case of cavities having negligible mode density around atomic frequency, spontaneous emission itself is smaller, and the pres- ence of a dc field shows significant inhibition or enhancement depending on whether the cavity is tuned below the atomic transition frequency or above the transition frequency.

G.S.A. thanks G. Rempe and H. Walther for discussions on this subject and C. Fabre for providing data on polarizabilities of Rydberg atoms.

\section{APPENDIX}

We outline how the time averaging is to be done. Let us consider the Schrödinger equation,

$$
\frac{\partial}{\partial t}|\psi(t)\rangle=-\frac{i}{\hbar} V(t)|\psi(t)\rangle
$$

where $V(t)$ consists of rapidly oscillating terms only, so that the time average of $V(t)$ is zero. Let $|\psi\rangle$ be written as

$$
|\psi\rangle=|\bar{\psi}\rangle+|\phi\rangle
$$

where $|\bar{\psi}\rangle$ is time-averaged part and $|\phi\rangle$ is the rapidly oscillating part. On substituting (A2) in (A1) we find that to the lowest order in $V(t)$,

$$
|\phi\rangle=-\frac{i}{\hbar} \int_{0}^{t} V(\tau) d \tau|\bar{\psi}\rangle,
$$

and

$$
\frac{\partial}{\partial t}|\bar{\psi}(t)\rangle=-\frac{i}{\hbar} \bar{V}(t)|\bar{\psi}\rangle
$$

where

$$
\bar{V}(t)=-\frac{i}{\hbar} \overline{V(t) \int_{0}^{t} V(\tau) d \tau .}
$$

The field-induced shift term in (9) is obtained by using Eq. (A5).
[1] W. Lange and H. Walther, Phys. Rev. A 48, 4551 (1993);G. S. Agarwal, W. Lange, and H. Walther, ibid. 48, 4555 (1993).

[2] W. Lange, H. Walther, and G. S. Agarwal, Phys. Rev. A 50, R3593 (1994).

[3] M. Macovei and C. H. Keitel, Phys. Rev. Lett. 91, 123601 (2003); J. Evers and C. H. Keitel, ibid. 89, 163601 (2002).

[4] G. S. Agarwal, M. O. Scully, and H. Walther, Phys. Rev. Lett. 86, 4271 (2001).

[5] G. S. Agarwal, Phys. Rev. A 54, R3734 (1996).

[6] H. R. Xia, C. Y. Ye, and S. Y. Zhu, Phys. Rev. Lett. 77, 1032 (1996);S. Y. Zhu and M. O. Scully, ibid. 76, 388 (1996).

[7] M. O. Scully and S.-Y. Zhu, Science 281, 1973 (1998).

[8] T. W. Mossberg and M. Lewenstein, in Cavity Quantum Electrodynamics, edited by P. R. Berman (Academic, New York, 1994), p. 171.

[9] P. Goy, J. M. Raimond, M. Gross, and S. Haroche, Phys. Rev.
Lett. 50, 1903 (1983); W. Jhe, A. Anderson, E. A. Hinds, D. Meschede, L. Moi, and S. Haroche, ibid. 58, 666 (1987).

[10] D. J. Heinzen, J. J. Childs, J. E. Thomas, and M. S. Feld, Phys. Rev. Lett. 58, 1320 (1987); D. J. Heinzen and M. S. Feld, ibid. 59, 2623 (1987).

[11] E. M. Purcell, Phys. Rev. 69, 681 (1946).

[12] D. Kleppner, Phys. Rev. Lett. 47, 233 (1981).

[13] C. Fabre, S. Haroche, and P. Goy, Phys. Rev. A 18, 229 (1978).

[14] T. F. Gallagher, Rep. Prog. Phys. 51, 143 (1988).

[15] Purcell's formula assumes that bad cavity is such that cavity mode remains well defined.

[16] W. P. Spencer, A. G. Vaidyanathan, D. Kleppner, and T. W. Ducas, Phys. Rev. A 24, 2513 (1981), present measurements of the lifetimes of sodium Rydberg states. 